\title{
JANVIER-FÉVRIER 1987
}

69e ANNÉE - N 763

\section{revue internationale de la croix-rouge}

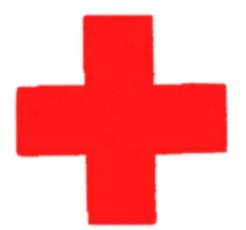

INTER ARMA CARITAS

GENĖVE

COMITÉ INTERNATIONAL DE LA CROIX-ROUGE FONDÉ EN 1863 


\title{
COMITÉ INTERNATIONAL DE LA CROIX-ROUGE
}

M. ALEXANDRE HAY, Docteur honoris causa des Universités de Genève et de Saint-Gall. avocat, ancien directeur général de la Banque nationale suisse, président (membre depuis 1975)

$\mathbf{M}^{\text {me }}$ DENISE BINDSCHEDLER-ROBERT, docteur en droit, professeur honoraire de l'Institut universitaire de hautes études internationales, Genève, juge à la Cour européenne des droits de l'homme, vice-présidente (1967)

MM. MAURICE AUBERT, docteur en droit, vice-président (1979)

ULRICH MIDDENDORP, docteur en médecine, chef de la clinique chirurgicale de l'Hôpital cantonal, Winterthour (1973)

RICHARD PESTALOZZI, docteur en droit, ancien vice-président du CICR (1977)

ATHOS GALLINO, docteur en médecine, maire de Bellinzone (1977)

ROBERT KOHLER, docteur ès sciences économiques (1977)

RUDOLF JÄCKLI, docteur ès sciences (1979)

DIETRICH SCHINDLER, docteur en droit, professeur à l'Université de Zurich (1961$1973 ; 1980$ )

HANS HAUG, docteur en droit, professeur honoraire de l'Ecole des hautes études économiques et sociales de Saint-Gall, ancien président de la Croix-Rouge suisse (1983)

PETER ARBENZ, licencié ès sciences politiques (1983), (en congé depuis mars 1986:

PIERRE KELLER, docteur en philosophie en relations internationales (Yale), banquier (1984)

RAYMOND R. PROBST, docteur en droit, ancien ambassadeur de Suisse, ancien secrétaire d'Etat au Département fédéral des Affaires étrangères à Berne (1984)

ODILO GUNTERN, docteur en droit, ancien député au Conseil des Etats (1985)

ANDRÉ GHELFI, ancien secrétaire central et vice-président de la Fédération suisse des travailleurs de la métallurgie (1985)

$\mathrm{M}^{\text {me }}$ RENÉE GUISAN, secrétaire générale de l" «Institut de la Vie» international, membre de la fondation suisse Pro Senectute, membre de l'«International Association for Volunteer Effort" (1986)

MM. DANIEL FREI, professeur de science politique à l'Université de Zurich (1986)

ALAIN B. ROSSIER, docteur en médecine, ancien professeur de rééducation des paraplégiques à l'Université de Harvard, privat-docent à la faculté de médecine de l'Université de Genève, président de la Société médicale internationale de paraplégie (1986)

CORNELIO SOMMARUGA, docteur en droit de l'Université de Zurich. Dr h.c. rer. pol. de l'Université de Fribourg (Suisse) (1986).

\section{CONSEIL EXÉCUTIF}

\author{
M. ALEXANDRE HAY, président \\ M. MAURICE AUBERT \\ M. RICHARD PESTALOZZI \\ M. ATHOS GALLINO \\ M. RUDOLF JÄCKLI \\ M. PIERRE KELLER \\ M. ANDRÉ GHELFI
}

Le Comité international de la Croix-Rouge (CICR) est, avec la Ligue des Sociétés de la Croix-Rouge et du Croissant-Rouge et les 144 Sociétés nationales de la Croix-Rouge et du Croissant-Rouge reconnues, l'une des trois composantes du Mouvement international de la Croix-Rouge et du Croissant-Rouge.

Institution humanitaire indépendante, le CICR est l'organe fondateur de la Croix-Rouge. Intermédiaire neutre en cas de conflits armés et de troubles, il s'efforce d'assurer, de sa propre initiative ou en se fondant sur les Conventions de Genève, protection et assistance aux victimes des guerres internationales et civiles, des troubles et tensions internes, apportant ainsi sa contribution à la paix dans le monde. 


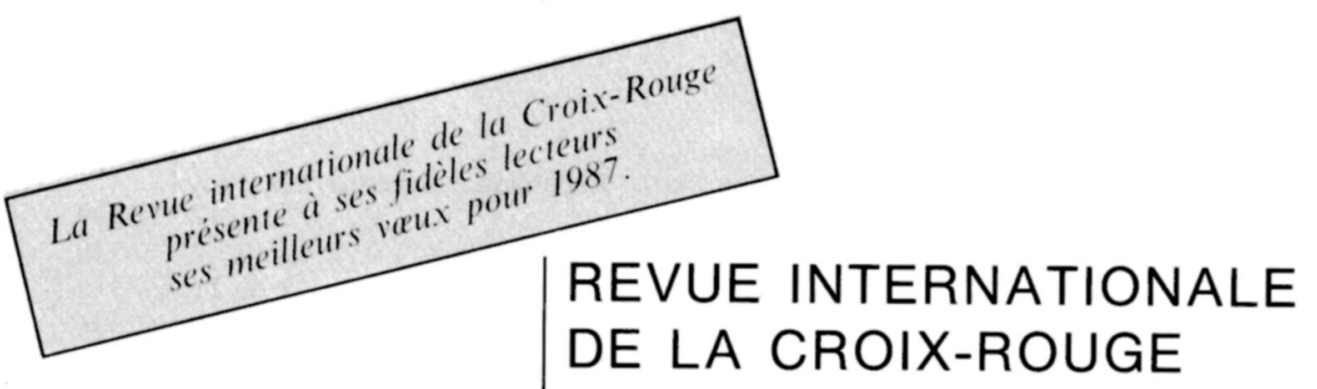

\section{COMITÉ INTERNATIONAL} DE LA CROIX-ROUGE

\section{DANS LE MONDE DE LA CROIX-ROUGE ET DU CROISSANT-ROUGE}

\section{REVUE INTERNATIONALE DE LA CROIX-ROUGE}

ISSN 0035-336]

\section{JANVIER-FÉVRIER $1987-\mathrm{N}^{\circ} 763$}

De quelques grandes questions humanitaires traitées par la $X X V^{e}$ Conférence internationale de la CroixRouge

Marco Sassòli: Le Bureau national de renseignements en faveur des victimes de conflits armés.

STATUTS ET REGLEMENT DU MOUVEMENT

INTERNATIONAL DE LA CROIX-ROUGE ET

DU CROISSANT-ROUGE

- Statuts du Mouvement international de la

Croix-Rouge et du Croissant-Rouge . . . . . . .

- Règlement du Mouvement international de la Croix-Rouge et du Croissant-Rouge . . . . . . .

Respect du droit international humanitaire - Rapport d'activité du CICR

Reconnaissance de la Société de la Croix-Rouge de Sainte-Lucie

Reconnaissance de la Société de la Croix-Rouge guinéenne

Reconnaissance de la Société de la Croix-Rouge angolaise

Reconnaissance de la Société de la Croix-Rouge du Suriname ................

Reconnaissance de la Société du Croissant-Rouge de Djibouti .................. 89

Récentes missions du Président du CICR . . . . . 91

Nomination de deux nouveaux membres honoraires . 92

Activités extérieures:

Afrique - Amérique latine - Asie - MoyenOrient -- Europe

Journée mondiale de la Croix-Rouge et du Croissant-Rouge 1987

Activités des Sociétés nationales de la Croix-Rouge et du Croissant-Rouge

La Croix-Rouge espagnole et «les marginaux» . 103

Diffusion du droit international humanitaire . . . 106

Second Séminaire arabe de droit international humanitaire . . . . . . . . . . . . . $V^{e}$ Séminaire régional africain sur le droit international humanitaire

Disparition d'un grand promoteur du message de Henry-Dunant 
Adhésion de la République argentine aux Protocoles .................. . 110

Déclarations interprétatives ......... 110

Adhésion de la République des Philippines au Protocole II

Etats parties aux Conventions de Genève du 12 août 1949 - Etats parties aux Protocoles du 8 juin 1977 (au 31 décembre 1986) . . . . . . . . . . . .

Résolution de l'Organisation des Etats Américains

Résolution de la $41^{\mathrm{e}}$ session de l'Assemblée générale des Nations Unies sur les Protocoles additionnels aux Conventions de Genève ......... .

\section{LIVRES ET REVUES}

Commentaire des Protocoles additionnels du 8 juin 1977 aux Conventions de Genève du 12 août $1949 \ldots \ldots \ldots \ldots \ldots \ldots . \ldots \ldots$

Mirador - my term as Hitler's guest (John Bur-

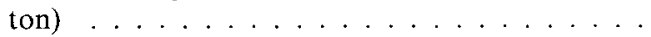

Ces lieux où Henry Dunant... (Roger Durand/Michel Rouèche) . . . . . . . . . . . . 126

Dans les Revues . . . . . . . . . . . . 127

Nouvelles publications ............. . 130

La Revue internationale de la Croix-Rouge est publiée par le Comité international de la Croix-Rouge (CICR) depuis 1869. Elle a porté à l'origine le titre de «Bulletin international des Sociétés de secours aux militaires blessés», puis de «Bulletin international des Sociétés de la Croix-Rouge».

- Organe officiel du CICR et du Mouvement international de la Croix-Rouge et du Croissant-Rouge,

- publication spécialisée dans le droit international humanitaire et la doctrine du CICR,

- chronique des activités internationales de la Croix-Rouge, ouvrage de référence essentiellement, qui garde la mémoire des événements,

la Revue internationale maintient un courant d'informations et constitue le lien nécessaire entre les membres du Mouvement international de la Croix-Rouge et du Croissant-Rouge.

RÉdACtION: Jacques Meurant, $D^{r}$ sc. pol, rédacteur en chef

ADresSE: $\quad$ Revue internationale de la Croix-Rouge

17 , avenue de la Paix

$\mathrm{CH}$ - 1202 - Genève, Suisse

ABONNEMENTS: un an 30 francs suisses; le numéro 5 francs.

Compte de chèques postaux: 12 - 1767 Genève

Compte bancaire: 129.986 Société de Banque Suissc, Genc̀ve

Seuls les textes signés par le Comité international de la Croix-Rouge engagent la responsabilité de celui-ci. 